\title{
Efficiency enhancement in a light-emitting diode with a two-dimensional surface grating photonic crystal
}

\author{
Hiroyuki Ichikawa and Toshihiko Baba ${ }^{\mathrm{a}}$ \\ Yokohama National University, Department of Electrical and Computer Engineering, 79-5 Tokiwadai, \\ Hodogayaku, Yokohama 240-8501, Japan
}

(Received 12 March 2002; accepted 21 November 2003)

\begin{abstract}
We demonstrate a light-emitting diode exhibiting 1.7-2.7-fold enhancement in light extraction efficiency compared with that for a planer device. It has a two-dimensional surface grating photonic crystal, which diffracts internal light with a large solid angle into the escape light cone. Due to its shallow grating separated from the active layer and micron order lattice constant, the fabrication process is simple, applicable to arbitrary semiconductor devices, and free from process-induced nonradiative recombinations. (C) 2004 American Institute of Physics. [DOI: 10.1063/1.1644033]
\end{abstract}

In a standard planar light emitting diode (LED), the efficiency is limited to several percents by a low light extraction efficiency $\eta_{\mathrm{ex}}$ due to the total internal reflection (TIR) at semiconductor/air boundaries. To avoid this problem, processes of epitaxial wafers into particular shapes have been developed. In general, however, such processes are complicated and not suitable for low cost mass production. A thin slab active layer sandwiched by low index claddings has been studied for the same purpose. It strongly confines light into the slab, and enhances various effects, e.g., the photon recycling, ${ }^{1,2}$ the scattering by a rough surface, ${ }^{3}$ the diffraction by a two-dimensional (2D) photonic crystal (PC) grating, ${ }^{4-6}$ and the plasmon-enhanced emission by a metal PC. ${ }^{7}$ However, the formation of electrodes is essentially difficult, since highly reflective boundaries are necessary for the enhancement. The process damage and surface recombination induced into the active layer are other problems. We demonstrated $>20$-fold enhancement in $\eta_{\mathrm{ex}}$ of a 2D PC of microcolumns. ${ }^{8,9}$ However, the internal efficiency was evaluated to be as low as $\sim 20 \%$ due to the surface recombination. In this study, we focus on a structure whose schematic is proposed by Kish et al. ${ }^{10}$ It is a $2 \mathrm{D}$ surface grating $\mathrm{PC}^{11}$ absolutely separated from the active layer, as shown in Fig. 1. Since only a shallow etching of the epitaxial wafer is required, the nonradiative effects are negligible. But $\eta_{\mathrm{ex}}$ can be improved by the conversion of $\boldsymbol{k}$ vector of the free propagating internal light.

For a bulk semiconductor with an index $n=3.17$ and air with an index $n_{0}=1.0$, the critical angle of the TIR, $\theta_{\mathrm{c}}$, measured from normal to the boundary is $18.4^{\circ}$. At each plane of a rectangular semiconductor, $\eta_{\mathrm{ex}}=(1 / 2) \Sigma_{s, p} \int_{0}^{\theta_{\mathrm{c}}}[1$ $-R(\theta)](\sin \theta / 2) \cdot d \theta \cong 1.8 \%$, where $R(\theta)$ is the angle- and polarization-dependent Fresnel reflection. For a double heterostructure and/or quantum-well wafer, this value will be slightly lower due to the waveguide effect of the active layer, but still of the same order. In the structure of Fig. 1, free propagating internal light reaches the grating, and the tangential component of the $\boldsymbol{k}$ vector, $\boldsymbol{k}_{\|}$, is changed by the grating $\boldsymbol{K}$ vector. As a result, the light angle is changed so that it can be extracted to air. The internal light occupies a larger solid angle than that of the narrow light cone for light extraction. Therefore, the change of light angle improves $\eta_{\mathrm{ex}}$ by the enhancement factor $F=\frac{1}{2} \int_{\theta_{\mathrm{c}}-\theta_{\mathrm{g}}}^{\theta_{\mathrm{g}}+\theta_{\mathrm{gif}}} \sin \theta d \theta$, where $\theta_{\mathrm{g}}\left(>\theta_{\mathrm{c}}\right)$ is the change of light angle by the grating and $\eta_{\text {dif }}$ is the diffraction efficiency. If $\eta_{\mathrm{dif}}=100 \%$, the maximum $\eta_{\text {ex }}$ would be $30 \%$ at $\theta_{\mathrm{g}}=90^{\circ}-\theta_{\mathrm{c}}=72^{\circ}$, which corresponds to $F=11.5$. However, actual $\eta_{\text {dif }}$ cannot be $100 \%$.

An actual 2D grating should have a triangular lattice with the hexagonal Brillouin zone (BZ) to minimize the anisotropy of the $\boldsymbol{K}$ vector. Figure 2 shows BZs for various lattice constant $a$, dispersion curves for the in-plane $\boldsymbol{k}$ vector in air, $\boldsymbol{k}_{0 \|}$, and in the semiconductor, $\boldsymbol{k}_{\|}$, and the zone-folded dispersion curve of $\boldsymbol{k}_{\|}$. In the BZ, $K_{\Gamma-X}$ and $K_{\Gamma-J}$ are $2 \pi / \sqrt{3} a$ and $4 \pi / 3 a$, respectively. Radii of dispersion curves $k_{0 \|}$ and $k_{\|}$are $2 \pi n_{0} / \lambda$ and $2 \pi n / \lambda$ for emitted wavelength $\lambda$, respectively. When $2 K_{\Gamma-X}>k_{0 \|}+k_{\|} \quad[a / \lambda<2 / \sqrt{3}(n+1)$ $=0.28$ for $n=3.17]$, the zone-folded curve does not enter the air curve, so the diffraction does not occur. When the BZ is smaller ( $a$ is larger) than this value, some amount of diffraction occurs. When the $\mathrm{BZ}$ is so small that it satisfies $K_{\Gamma-J}$ $\leqslant k_{0 \|}(a / \lambda \geqslant 2 / 3)$, the $\mathrm{BZ}$ is wholly included in the air curve, and this gives the maximum $\eta_{\text {dif }}$. However, $\eta_{\text {dif }}$ cannot be unity for such a small BZ, since light can find not only the extracted light cone but also another solid angle not extracted by the diffraction. Even in light diffracted into the extracted light cone, half goes downward. Therefore, $\eta_{\text {dif }}$ is decreased to $\sim 25 \%$, and finally $F \sim 3$ is expected.

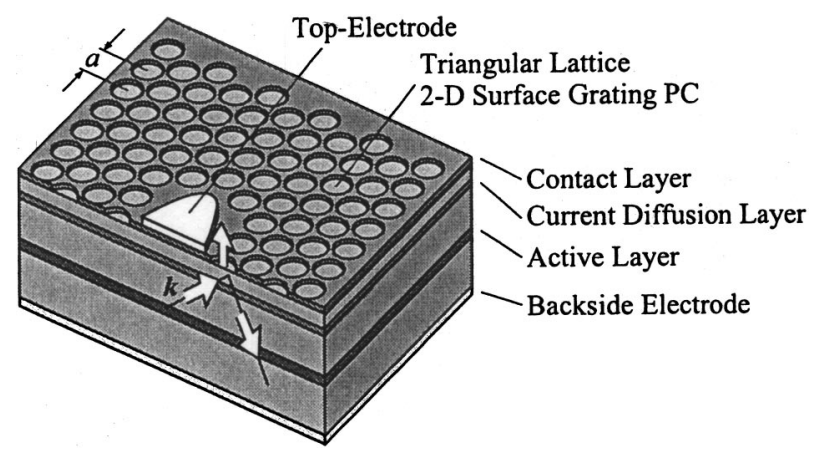

FIG. 1. Schematic of 2D LED with surface grating PC.

${ }^{a)}$ Electronic mail: baba@ynu.ac.jp 


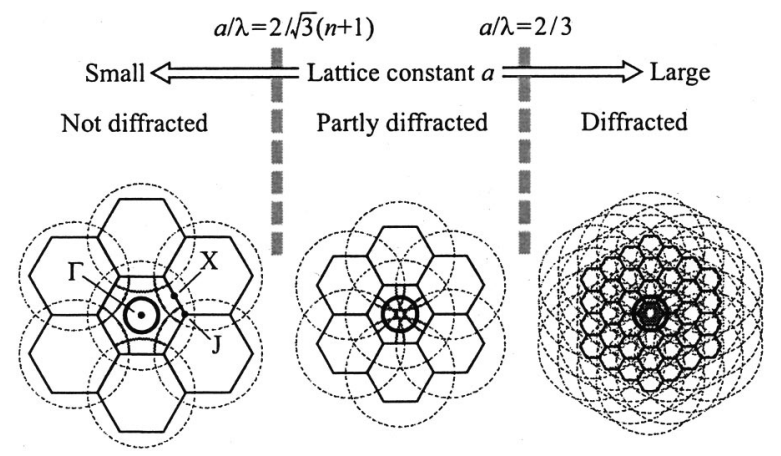

FIG. 2. Brillouin zones for 2D triangular lattice, dispersion curves of $k_{0 \|}$ (center thick black circle) and $k_{\|}$(dotted gray circle), and zone-folded dispersion curves of $k_{\|}$(gray circle).

For quantitative estimations, we simulated light propagation and diffraction using the finite difference time domain (FDTD) method. A complete three-dimensional (3D) calculation is difficult, since for sufficient grating periodicity and calculation accuracy, a 3D model of over $10^{8}$ Yee cells will be required, which roughly corresponds to 10 GB computer memory. For simplicity, we employed a 2D model of 20 $\times 4.8 \mu \mathrm{m}^{2}$ uniform bulk semiconductor having a onedimensional (1D) rectangular surface grating with a depth of $0.6 \mu \mathrm{m}$ and $a=0-2 \mu \mathrm{m}$. A Gaussian beam of $3.8 \mu \mathrm{m}$ in $1 / e^{2}$ width propagating by an angle $\theta$ of $0^{\circ}-70^{\circ}$ was continuously excited inside the semiconductor for $s$ or $p$ polarization at $\lambda=1.55 \mu \mathrm{m}$. Light output by one-time diffraction was observed for each angle $\theta$ just above the grating. We evaluated $\eta_{\mathrm{ex}}$ by integrating this output multiplied by the factor of the solid angle effect with respect to $\theta$. The $F$ factor was obtained by comparing $\eta_{\mathrm{ex}}$ with that for the planar device. As shown in Fig. 3, $F$ increases for $a>0.4 \mu \mathrm{m}(a / \lambda$ $>0.258$ ), and gets close to its maximum at $a=0.8 \mu \mathrm{m}$ $(a / \lambda=0.516)$. If we apply the earlier discussion on dispersion characteristics to the $1 \mathrm{D}$ grating, we notice that the zone-folded curve enters the air curve for $a / \lambda>0.239$ and the $\mathrm{BZ}$ is included in the air curve for $a / \lambda>0.503$. Thus, the discussion and the result of Fig. 3 are consistent with each other. The maximum $F$ are 3.1 and 3.5 for $p$ and $s$ polarizations, respectively, for $a=1.6 \mu \mathrm{m}$. In actual LEDs, dif-

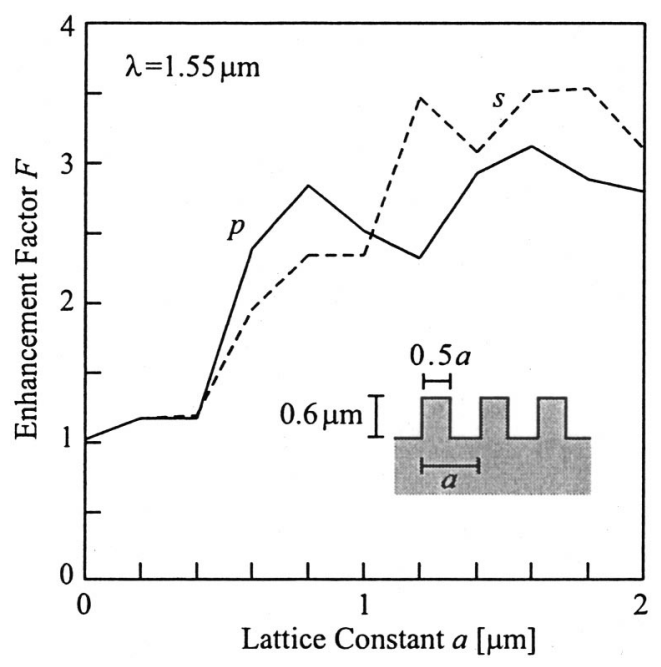

FIG. 3. Enhancement factor $F$ of light extraction efficiency calculated with

the diffusion of internal light, the diffusion length of carriers
lattice constant $a$.
Downloaded 21 Jan 2004 to 133.34.23.211. Redistribution subject to AlP license or copyright, see http://ojps.aip.org/aplo/aplcr.jsp

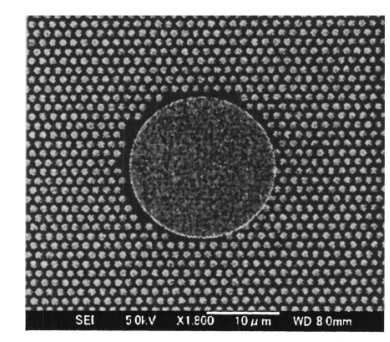

(a)

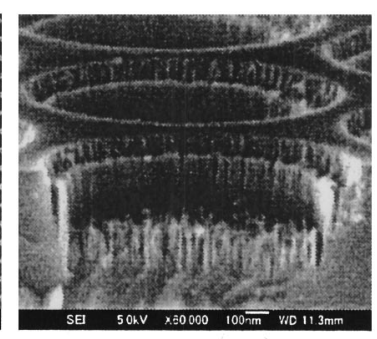

(b)
FIG. 4. Scanning electron micrographs of (a) center AuZn electrode surrounded by triangular lattice $\mathrm{PC}$, and (b) airholes with many nanometer-size projections on the bottom.

fracted light going downward is partly reflected by a backside electrode and extracted to upper direction, although the reflectivity is no higher than $80 \%$ for any metals after annealing. Therefore, $F$ will be slightly higher than those in Fig. 3. The FDTD simulation also showed that $F$ takes its maximum when the hole filling factor $f$ of the grating is $0.6-0.7$, but that the characteristic slowly changes with $f$ due to the strong coupling coefficient of the grating having high index contrast boundaries. This suggests a large fabrication tolerance of the grating.

In the experiment, we prepared an epitaxial wafer with $n$-InP substrate, undoped GaInAsP active layer, $1.5-\mu \mathrm{m}$-thick $p$-InP layer, $0.2-\mu \mathrm{m}$-thick $p^{+}$-GaInAsP $\left(\lambda_{g}=1.3 \mu \mathrm{m}\right)$ current diffusion layer, $0.5-\mu \mathrm{m}$-thick $p^{+}$-InP layer, and $0.2-\mu \mathrm{m}$ thick $p^{+}$-GaInAsP $\left(\lambda_{g}=1.3 \mu \mathrm{m}\right)$ contact layer. The active layer was composed of 1-\% compressively strained five quantum wells (CS-QWs) whose photoluminescence wavelength was centered at $1.584 \mu \mathrm{m}$. The $20 \mu \mathrm{m} \phi$ top AuZn electrode was formed on the contact layer. Around this, triangular lattice airholes were formed by electron beam (EB) lithography and $\mathrm{Cl}_{2} / \mathrm{Xe}$ inductively coupled plasma etching. ${ }^{8,9}$ The etching was stopped in the middle of the InP layer beneath the contact layer. The substrate was lapped and polished to $\sim 100 \mu \mathrm{m}$ thickness. AuGe was evaporated on this side, and annealed to form the ohmic contact. As shown in Fig. 4, many nanometer-size projections were formed on the bottom of each airhole. These projections were expected to act as an antireflection (AR) coating against the Fresnel reflection. ${ }^{12}$ The FDTD simulation showed that it can increase the maximum $F$ by $7 \%$.

In the measurement, the continuous current was injected into a device at room temperature. The light output was detected by a $1 \mathrm{~cm} \phi$ Ge photodiode placed by $5 \mathrm{~mm}$ distant from the device top. This detecting condition covers $\sim 30 \%$ of $2 \pi$ steradians, but detectable light output is larger than this value because of the directional light radiation caused by the angle-dependent Fresnel reflection and diffraction efficiency. The FDTD simulation indicated that the earlier condition can detect $73 \%$ of the radiation pattern from the planar LED and 55\%-73\% from PC LEDs with various $a$, respectively. The inset of Fig. 5 shows the near-field pattern of light observed from the top. Since the top electrode shadowed the center part, light output was only obtained from outside of the electrode. In planar LEDs, the decay length of output intensity, which was measured from the edge of the electrode, was $\sim 8 \mu \mathrm{m}$. Considering this length and simulating the diffusion of internal light, the diffusion length of carriers 


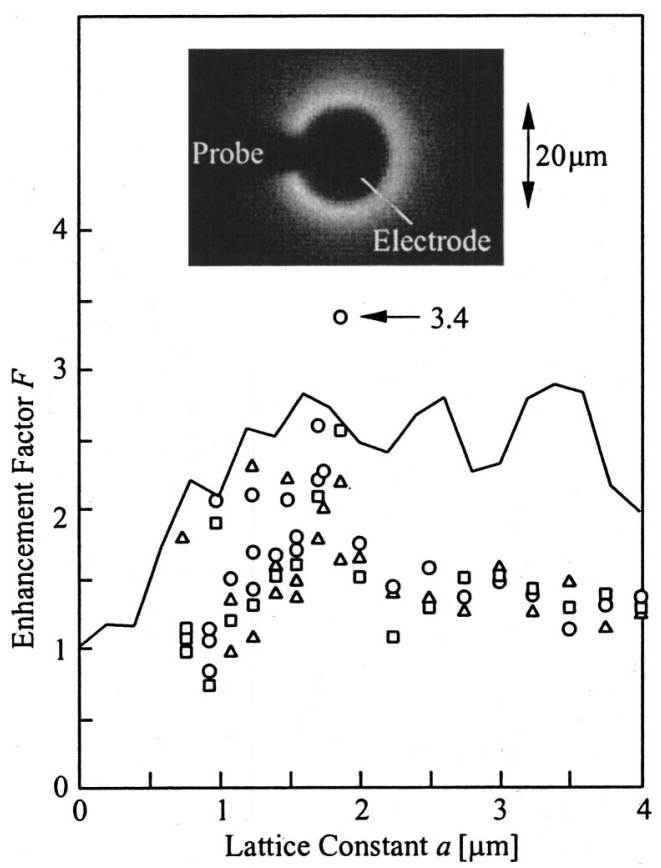

FIG. 5. Experimental plots and theoretical curve of enhancement factor $F$. Squares, circles, and triangles denote air filling factor of $0.2,0.4$, and 0.6 , respectively. Inset shows the near field pattern of light output.

was estimated to be $5 \mu \mathrm{m}$. For this length and the electrode size, the fraction of effective carriers for emission extracted in the air was estimated to be $20 \%$. An absolute value of differential quantum efficiency $\eta_{d}$ was measured to be $0.19 \%-0.22 \%$ for planar LEDs. This is a reasonable value, when considering the light extraction efficiency from one planar surface, the shadowing effect, and an internal efficiency of the wafer. Thus, all we can evaluate here is the relative enhancement factor $F$ by the PC grating.

To eliminate the influence of heat and Auger effects, $\eta_{d}$ was measured at near zero current and compared with that for the planar device. Figure 5 shows experimental plots and the theoretical curve of $F$ as a function of lattice constant $a$. Here, the theoretical curve was calculated for the mixed polarization by considering the electron to heavy-hole transition in the CS-QWs, which contributes to the in-plane polarization. Maximum $F$ was 3.4 for $a=1.86 \mu \mathrm{m}$ and $f$ $=0.4$. Even if we consider a backside reflection and the AR effect by the projections, this value is too high compared with the theoretical value, and looks peculiar compared with other plots. A reason considered for this is another AR effect arising from the phase shift at the grating step, which is satisfied by the filling factor $f$. A 3D FDTD calculation with a single shallow airhole model showed that such an AR effect can occur for light normally incident to the surface. Since this phenomenon has a relatively strong directionality, a large $F$ factor of 3.4 cannot be thought as an essential improvement of $\eta_{\mathrm{ex}}$. Neglecting this plot, $F=1.7-2.7$ for $a \sim 1.7 \mu \mathrm{m}$ is typical, which is slightly lower than the theoretical value. It can be explained by the different directionality of light radiation between the planar device and PC LEDs, as mentioned earlier.

Enhancement factors of 1.7-2.7 are smaller than those discussed for other PC LEDs. Still, we think that the 2D surface grating PC LED is attractive because of its very simple process and the applicability to arbitrary semiconductors and other emitting materials including organic ones. ${ }^{13-15}$ Since the maximum efficiency is obtained with micron-order lattice constant, the formation of the PC does not necessarily require an EB lithography but will be possible by photolithography, interference exposure, molding, wet etching, etc. The PC effect is still effective with a plastic mold, although a plastic mold of $n_{0}=1.5$ reduces the theoretical $F$ value to $\sim 2.0$ due to the improvement of $\eta_{\mathrm{ex}}$ of the planar device. The combination of the PC and a round shape plastic mold will give $F=4-5$. If a high reflectivity backside mirror, e.g., a multilayer with chirped thicknesses for a wide stopband, is added, $F=8-10$ will be possible.

This work was partly supported by the IT Program by the Ministry of Education, Science, Sports and Culture, and by CREST No. 530-13 of Japan Science and Technology Corporation.

${ }^{1}$ I. Schnitzer, E. Yablonovitch, C. Caneau, and T. J. Gmitter, Appl. Phys. Lett. 62, 131 (1993).

${ }^{2}$ T. Baba, R. Watanabe, K. Asano, F. Koyama, and K. Iga, Jpn. J. Appl. Phys., Part 1 35, 97 (1996).

${ }^{3}$ I. Schnitzer, E. Yablonovitch, C. Caneau, T. J. Gmitter, and A. Scherer, Appl. Phys. Lett. 63, 2174 (1993).

${ }^{4}$ S. Fan, P. R. Villeneuve, and J. D. Joannopoulos, Phys. Rev. Lett. 78, 3294 (1997).

${ }^{5}$ M. Boroditsky, R. Vrijen, T. F. Krauss, R. Coccioli, R. Bhat, and E. Yablonovitch, J. Lightwave Technol. 17, 2096 (1999).

${ }^{6}$ A. A. Erchak, D. J. Ripin, S. Fan, P. Rakich, J. D. Joannopoulos, E. P. Ippen, G. S. Petrich, and L. A. Kolodziejski, Appl. Phys. Lett. 78, 563 (2001).

${ }^{7}$ J. Vučković, M. Lončar, and A. Scherer, IEEE J. Quantum Electron. 36, 1131 (2000).

${ }^{8}$ T. Baba, K. Inoshita, H. Tanaka, J. Yonekura, M. Ariga, A. Matsutani, T. Miyamoto, F. Koyama, and K. Iga, J. Lightwave Technol. 17, 2113 (1999).

${ }^{9}$ H. Ichikawa, K. Inoshita, and T. Baba, Appl. Phys. Lett. 78, 2119 (2001).

${ }^{10}$ F. E. Kish, Jr. and M. R. Krames, US Patent No. 5,779,924 (1998).

${ }^{11}$ T. Baba and H. Ichikawa, Proc. Optoelectronic and Commun. Conf., 2002, p. 9C2-1.

${ }^{12}$ Y. Kanamori, H. Kikuta, and K. Hane, Jpn. J. Appl. Phys., Part 2 39, L735 (2000).

${ }^{13}$ T. Baba, K. Shiratsuchi and R. Nakamura, applied for Jpn. Patent No. 2002-188157 (2002).

${ }^{14}$ Y. J. Lee, S. H. Kim, J. Huh, G. H. Kim, Y. H. Lee, S. H. Cho, Y. C. Kim, and Y. R. Do, Appl Phys. Lett. 82, 3779 (2003).

${ }^{15}$ M. Fujita, T. Ueno, T. Asano, S. Noda, H. Ohhata, T. Tsuji, H. Nakada, and N. Shimoji, Electron. Lett. 39, 1750 (2003). 